\title{
Sex differences in the left and right hemifields of normal subjects with computerised static perimetry
}

\author{
Howard Cohn, Maria DeAgostini, Danièle Aron-Rosa, Laurent Laloum, François Boller
}

\begin{abstract}
Results of routine perimetric testing of the left and right hemifields in normal subjects have been assumed to be symmetric although asymmetry due to hemispheric dominance has been established for other psychophysical tests. These asymmetries have sometimes been related to sex. With the advent of computerised static perimetry, subtle differences between the left and the right hemifields might be found that were not obvious before. This study investigated differences in retinal sensitivity between the hemifields and the role of sex and eye dominance. Forty three unequivocally right handed and right eye dominant normal adult volunteers, 24 females and 19 males, underwent Humphrey 24-2 testing, half beginning with the left eye, the other half with the right eye. The Peridata program was used to calculate decibel totals per hemifield. Four subjects were excluded because of poor cooperation or test artefacts. In females, the total of the left hemifield was significantly less than the right $(p<0.01)$ by a mean 18.2 (SD 24) dB equivalent to a difference of $0.34 \mathrm{~dB}$ per tested point. No significant difference in hemifields was found for males, between the sexes for both eyes combined, or between the two eyes for either sex. It was concluded that asymmetries in retinal sensitivity with respect to the vertical axis may be physiological and found in females, but not in males.
\end{abstract}

(Brf Ophthalmol 1994; 78: 837-841)

Routine kinetic and static visual field tests have not been known to reveal left right asymmetries, and the left and the right hemivisual fields in normal persons have generally been assumed to be symmetrical. However, asymmetries have been established for cognitive tests involving the visual fields. These include visual reaction time, ${ }^{12}$ memory for colours, ${ }^{3}$ and perception of lightness ${ }^{4}$ as well as neurophysiological events such as visual evoked potentials. In general, studies reveal a right visual field superiority for verbal tasks and a left field superiority for nonverbal tasks. ${ }^{6}$ Some papers have related these asymmetries to sex and have generally found a right visual field superiority for verbal tasks in males. ${ }^{78}$ Although classic kinetic and static methods to date are not known to reveal any hemifield asymmetry, the quantitative measurements of computerised static threshold perimetry could reveal differences. This study was prompted by the following case history.

CASE HISTORY

A 28-year-old white woman was referred because of recurrent generalised headache unassociated with other symptoms. She gave no history of any migraine phenomenon. Visual acuity was $6 / 6$ uncorrected in each eye. A complete ophthalmic examination was entirely within normal limits. A Humphrey 24-2 computerised visual field test performed while the patient was free from headache was judged to be within normal limits (Fig 1) but showed several relative defects of borderline significance in the temporal field of the left eye and the nasal field of the right eye. Upon examination of the vertical meridian in each eye, some retinal sensitivities in the left hemifield appeared to be less than the corresponding points in the right hemifield. Inspection of the grey scales also suggested a slight left lateral hemianopic defect. A complete neurological evaluation, including computed tomography failed to reveal any abnormality. A diagnosis of tension headache was made. The question was raised whether a minor hemianopic difference might be physiological in some subjects.

This study investigated threshold differences between lateral hemifields in normal men and women.

\section{Material and methods}

Forty three normal volunteers with right eye, hand, and foot dominance underwent visual field testing with the Humphrey 24-2 program. Hand and foot preferences were determined by the following questions derived from a longer questionnaire': with which hand do you write, eat with a spoon, throw a ball, and brush your teeth? With which foot do you kick a ball? Eye dominance was determined by asking subjects to look at a distant object through a hole in a piece of paper held at arm's length. All subjects underwent a complete ophthalmic examination including visual acuity testing, external and slitlamp examination with intraocular pressure measurement, and dilated ocular fundus examination. No subject had a positive history of migraine or other vasospastic disorder. For inclusion into the study uncorrected or best corrected visual acuity was $6 / 6$ or better in both eyes with a maximum tolerated refractive error of plus or minus 2 dioptres spherical equivalent including a maximum of 1 dioptre cylinder and less than 1 dioptre anisometropia.

Visual field testing was performed with the Humphrey visual field analyser using the 24-2 test and age-appropriate add. Half the subjects began testing with the left eye and half with the right eye to reduce any possible differences between the two eyes as a result of learning or fatigue. No subjects had undergone a computerised visual field test before this study. Subjects provided consent, but were not told the exact 
purpose of the study before taking the visual field test.

After completion of the visual field test, four subjects were excluded because of obvious artefacts and/or poor cooperation defined by the standard Humphrey parameters. A total of 39 subjects were included. They were 23 females of mean age $40 \cdot 7(10 \cdot 1)$ years and 16 males of mean age $41 \cdot 2(10.9)$ years. The Peridata program (courtesy of Dr Jorg Weber, Cologne, Germany) was used to calculate the total retinal sensitivity in decibels per hemifield. For example, to calculate the right hemifield, the 28 points of the temporal field of the right eye were added to the
A

NAME DE.P

Stimulus III. White. bckgnd 31.5 ASB blind spot check size III Strategy full threshold
Left

Birthdate 02-08-60 Date 25-04-88

Fixation target Central $10 \quad$ Time 12:07:28

RX used +2 DS DCX DEG Pupil diameter 8.0 MM VA 1.0

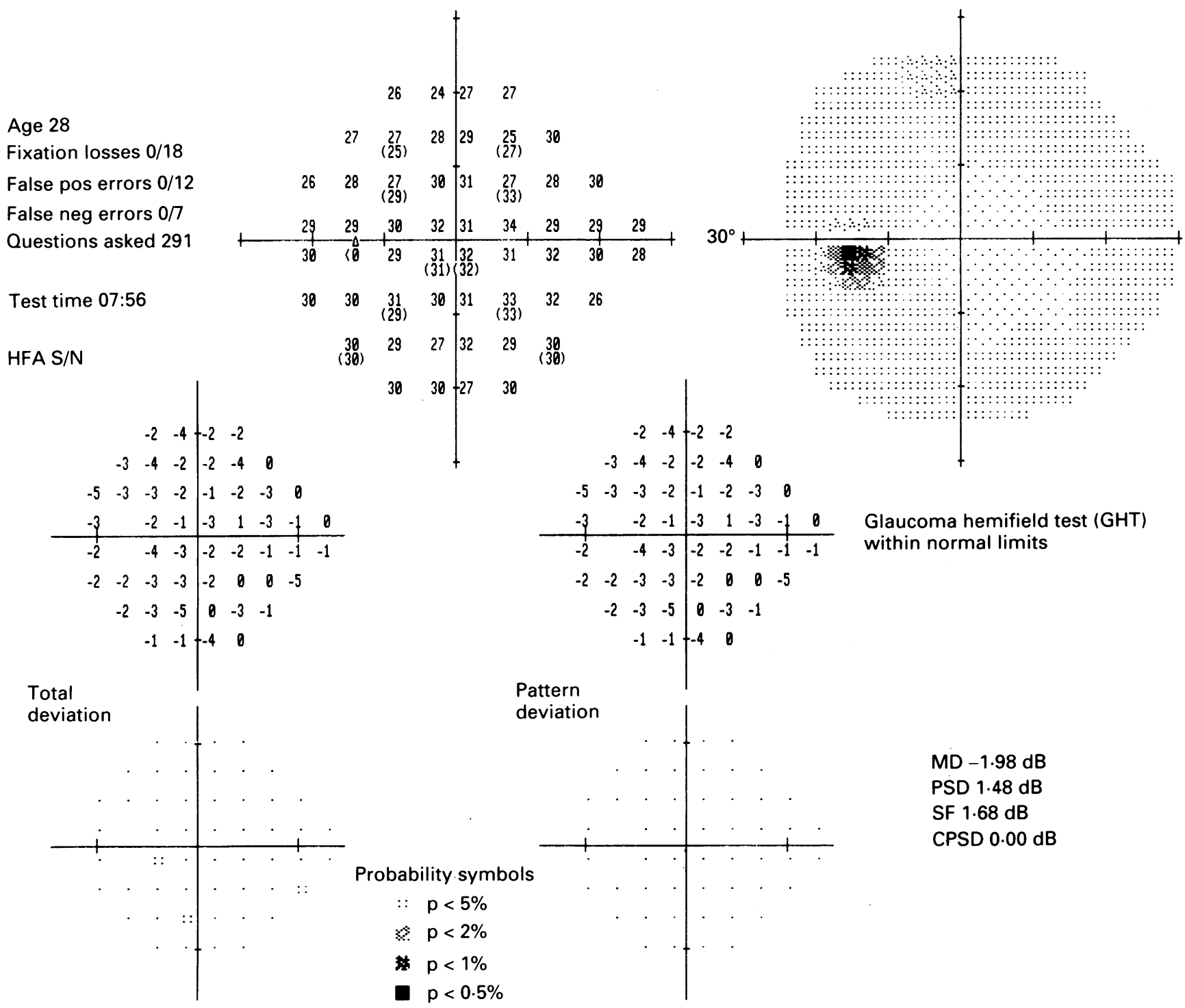

REV 9.2

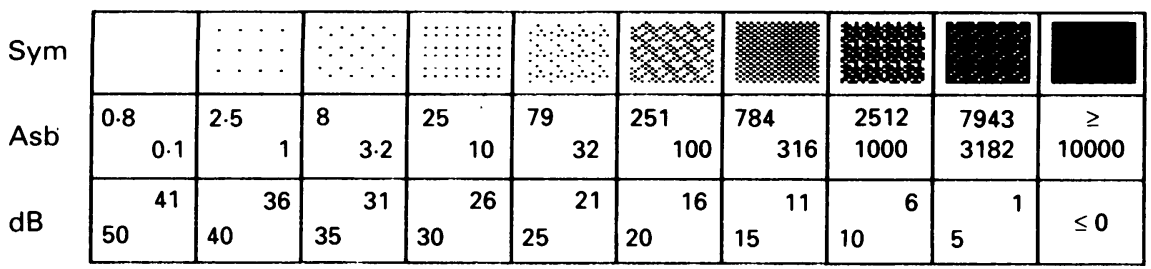

Figure 1 Case history: visual fields of a 28-year-old woman referred for ophthalmic examination. These fields were interpreted to be within normal limits, but comparison of corresponding points on either side of the vertical meridian suggested slightly decreased dB levels in the left hemifield of each eye. After a negative neurological evaluation, the question was raised whether a left lateral hemianopic difference might be physiological. 
26 points of the nasal field of the left eye. Twice thresholded points were taken as the average of the two values. In order to determine possible visual field asymmetries, the total $\mathrm{dB}$ for the right hemifield was subtracted from the left hemifield for each subject. The results were analysed separately for each subgroup (males and females), and then considering the 39 subjects as one group. Next, to see if eye dominance affects visual field results, the total score of the 54 test points of the left eye were compared with those of the right eye. Total thresholds of both eyes combined were also compared between the two sexes. Paired $t$ tests were used for each analysis.

\section{Results}

Table 1 shows the mean difference between the left and right hemifield $\mathrm{dB}$ scores in females and
NAME DE.P

Stimulus III. White.bckgnd 31.5 ASB blind spot check size III Strategy full threshold

Fixation losses $0 / 20$

False pos errors $1 / 5$

False neg errors $0 / 9$

Questions asked 345

Test time 10:19

HFA S/N
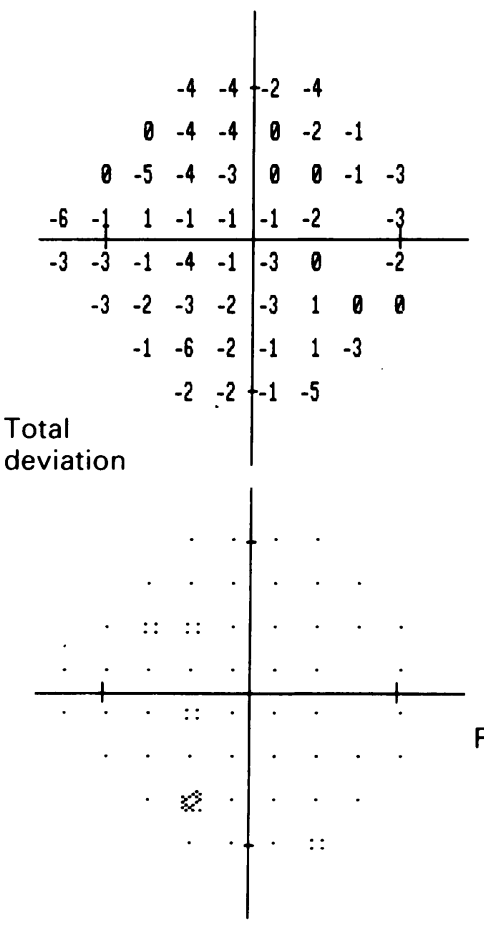

Probability symbols

$: \mathrm{p}<5 \%$

*. $p<2 \%$

$p<1 \%$

$p<0.5 \%$
Right

Birthdate 02-08-60 Date 25-04-88

\begin{tabular}{|c|c|}
\hline & Central \\
\hline$R X$ used +2 DS & DCX \\
\hline
\end{tabular}
RX used +2 DS DCX DEG Pupil diameter 8.0 MM VA 1.0 
Figure 2 Scatter plots showing the difference between the left and right hemifields as a function of total retinal sensitivity for 23 females $(A)$ and 16 males $(B)$. Most female data points are negative indicating a significant decrease in retinal sensitivity for the left hemifield. For males, the data points of hemifield comparison are more evenly distributed.

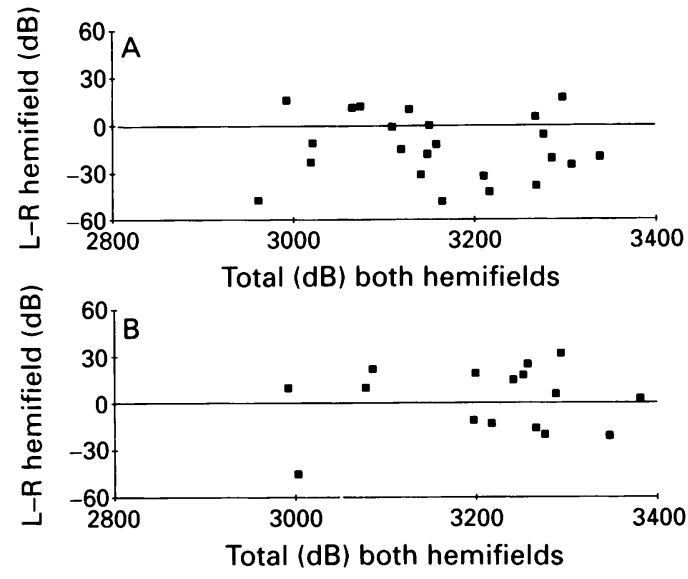

males. In females, the left hemifield total $\mathrm{dB}$ was significantly $(\mathrm{p}<0.01)$ inferior to the right (mean (SD) $18(23.4)$ or $0.34 \mathrm{~dB}$ per tested point). For males, the difference was only plus 0.9 (20) $\mathrm{dB}$ (not significant). Results of males and females taken together (Table 1) showed no significant differences between the hemifields (mean difference minus $10(22) \mathrm{dB}$ ). Comparison of $\mathrm{dB}$ totals between the two eyes (Table 2) revealed no significant difference for males or females. Comparison of $\mathrm{dB}$ scores of both eyes combined between females and males showed no significant difference. It was 3157 (112) dB for females and $3212(112) \mathrm{dB}$ for males $(t=1 \cdot 47)$.

Figure 2 presents scatter plots of the results of individual subjects showing the difference between the left and right hemifields as a function of total retinal sensitivity. As can be seen, most of the female data points (Fig 2A) are negative, indicating the significant decrease in retinal sensitivity found in the left hemifield of females. Figure 2A shows that not all females in the present study had decreased retinal sensitivity in the left hemifield; of the 23 females included in the final sample, six $(26 \%)$ did not. For males (Fig 2B), the results are more evenly distributed. Out of the 16 males, six (37\%) had a decrease in the left hemifield while $10(63 \%)$ had a relative decrease in the right hemifield with no significant overall difference between hemifields.

Table 1 Comparison of mean (SD) decibel (dB) totals of the left and right hemifields of both eyes combined in right handed subjects. Each total represents the mean sum of the 54 thresholded points combining the temporal field of one eye with the nasal field of the other. Twice measured points are taken as the average. (The negative difference in females indicates the left hemifield has a significantly lower measured retinal sensitivity $(p<0 \cdot 01)$. In males there is no significant difference)

\begin{tabular}{lllll}
\hline & $\begin{array}{l}\text { Left field } \\
(d B)\end{array}$ & $\begin{array}{l}\text { Right field } \\
(d B)\end{array}$ & $\begin{array}{l}\text { Left-right } \\
(d B)\end{array}$ & Significance \\
\hline $\begin{array}{llll}\text { Females }(\mathrm{n}=23) \\
\text { Males }(\mathrm{n}=16)\end{array}$ & $1570(59)$ & $1588(55)$ & $-18(24)$ & $\mathrm{p}<0.01$ \\
& $1607(58)$ & $1606(56)$ & $+0.9(20)$ & $\mathrm{NS}$ \\
\hline
\end{tabular}

Table 2 Comparison of retinal sensitivities between the two eyes in right eye dominant subjects. Each total is the mean (SD) sum of the 54 thresholded points per eye. (No significant difference was found for either sex)

\begin{tabular}{lllll}
\hline & $\begin{array}{l}\text { Left eye } \\
(d B)\end{array}$ & $\begin{array}{l}\text { Right eye } \\
(d B)\end{array}$ & $\begin{array}{l}\text { Left-right } \\
(d B)\end{array}$ & Significance \\
\hline $\begin{array}{l}\text { Females } \\
\text { Males }\end{array}$ & $\begin{array}{l}1578 \cdot 5(69) \\
1603(58)\end{array}$ & $1579(50)$ & $-0 \cdot 5(45)$ & NS \\
\hline
\end{tabular}

\section{Discussion}

This study shows a significant difference in retinal sensitivity in favour of the right hemifield of normal right handed females. This is the first report of a physiological hemifield asymmetry in perimetric testing. The lateral hemifield difference is not significant when the results of females and males are combined. This probably explains why normal perimetric data are not known to demonstrate lateral differences.

How might one explain the present results? Testing of a visual field point by the static method is a complex psychophysical test beginning with physical action of light stimuli at the retinal level, leading to cortical perception and intracortical associations to the motor area resulting in the voluntary hand movement of pressing on a button. The present finding of asymmetry in retinal sensitivity may be due to a real difference in retinocortical perception. Alternatively, it has been proposed that asymmetry of the gross anatomy of the cortex ${ }^{10}$ is correlated with hemispheric dominance, especially for the occipital lobes. "On the other hand, it may be that only higher processing beyond detection is affected by cortical functional asymmetry.

For over 130 years, findings concerning hemispheric asymmetries have led the scientific world to discuss first cerebral dominance and more recently hemispheric specialisation. ${ }^{12}$ Asymmetry has been well established in the performance of several visual tests. In right handed individuals, manual and vocal reaction times to visual stimuli are shorter in the right visual field. ${ }^{13}$ A right visual field superiority has also been demonstrated for colour memory ${ }^{3}$ and for perception of lightness. ${ }^{+}$A very recent paper ${ }^{1+}$ has confirmed other findings demonstrating larger hemifield superiority for verbal tasks and left field advantage for non-verbal tasks. It is only in the past 30 years $^{1516}$ that the issue of possible sex related differences has emerged in the literature. This has involved not only functional but also anatomical asymmetries. ${ }^{17} 18$

Most studies showing sex related asymmetric results demonstrate more asymmetry for males. ${ }^{16}{ }^{19}$ However, some studies do show more asymmetry for females. These studies are generally concerned with motor rather than cognitive aspects of behaviour; in particular, a sex difference has been found in studies of handedness ${ }^{20}$ and of manual skills. ${ }^{21}{ }^{22}$ These studies indicate that asymmetry for hand preference and hand skills (more women being strongly right handed and more skilled with their right hand) appear greater among females than among males. The findings of the present study showing a right hemifield superiority for women go in the same direction.

In interpreting our results, one might consider the question of whether the hand in which the subject holds the button could affect visual field test results. The present study did not address this question. Since the button was located on the right side of the apparatus, our subjects (all of whom were right handed) invariably held the button in their right hand.

Precision of the present study was not considered to be changed by including the blind 
spot. There were minor decibel differences between right and left blind spot values that appear equally distributed between the two eyes.

Eye dominance does not apparently affect retinal sensitivity for either sex as shown in Table 2 , although it should be noted that foveal testing is not included in the present study. If there is a comparable level of asymmetry between the left and the right hemifield of each eye, one would expect no significant difference between the total $\mathrm{dB}$ values of the two eyes. In addition, since the data shown in Tables 1 and 2 suggest a slightly decreased $\mathrm{dB}$ total for females, we performed a comparison of overall retinal sensitivity between sexes for both eyes combined. This comparison yielded no significant difference.

In view of the present study, one may want to take a new look at the interpretation of studies finding sex differences, for example with tachistoscopic methods, ${ }^{6}$ where the inferior sensitivity of the left visual field in women may have influenced the results.

It is concluded that differences in threshold values across the vertical meridian with lower sensitivities in the left hemifield are likely to be physiological in females, in the absence of supporting neurological signs or symptoms. This finding may be of help to clinicians and researchers in the interpretation of computerised visual field tests. There are computer programs such as STATPAC II (Zeiss-Humphrey) with the glaucoma hemifield test designed to show subtle hemifield differences. The program analyses matching areas where glaucomatous defects often begin on either side of the horizontal meridian and provides an index of suspicion for glaucomatous damage. A similar program analysing differences across the vertical meridian might be useful in neurological studies.

The result of this study have implications for use of age related normal data bases. If one considers females and males separately, more precise comparison with normal values should be obtained. It is of interest to note that inquiries to confirm the findings of this study with the existing Humphrey and Octopus normal data bases revealed that females are not distinguished from males in either system. Furthermore, this information is no longer available since data base subjects are now identified only by age and number. Even though the results of the present study are statistically significant, further larger scale studies should be performed.

Presented at the annual meeting of the Association for Research in Presented at the annual meeting of the Association for Res
Vision and Ophthalmology, Sarasota, Florida, May 1991.

1 Rizzolatti G, Umiltà C, Berlucchi G. Opposite superiorities of the right and left cerebral hemispheres in discriminative reaction time to physiognomical and alphabetical material. Brain 1971; 94: 431-42.

2 Corballis M, Manalo R. Effect of spatial attention on mental rotation. Neuropsychologia 1993; 31: 199-205.

3 Malone D, Hannay HJ. Hemispheric dominance and normal color memory. Neuropsychologia 1978; 16: 51-9.

4 Davidoff JB. Hemispheric differences in the perception of lightness. Neuropsychologia 1975; 13: 121-4.

5 Klem W, Gibbons WD, Allen RG, Richey EO. Hemispheric lateralization and handedness correlation of human evoked 'steady-state' responses to patterned visual stimuli. Physiol Psychol 1980; 8: 409-16.

6 Bryden MP. Laterality. Functional asymmetry in the intact brain. New York: Academic Press, 1982.

7 Bradshaw GL, Gates EA. Visual field differences in verbal tasks: effects of task familiarity and sex of subjects. Brain and Language 1978; 5: 166-87.

8 Martin CM. Verbal and spatial encoding of visual stimuli: the effects of sex, hemisphere and yes-no judgements. Cortex 1978; 14: 227-33.

9 Dellatolas G, De Agostini M, Jallon P, Poncet M, Rey M, Lellouch J. Mesure de la préférence mannuelle par autoquestionnaire dans la population française adulte. Revue de Psychologie Appliquée 1988; 38: 117-36.

10 Geschwind N, Levitsky W. Human brain: left-right asymmetries in temporal speech regions. Science 1968; 161: 186-7.

11 LeMay M. Radiological, developmental and fossil asymmetries. In: Geschwind N, Galaburda AM, eds. Cerebral dominance. The biological foundations.

12 Bryden $M$. Cerebral specialization: clinical and experimental assessment. In: Boller F, Grafman J, eds. Handbook of neuropsychology. Amsterdam: Elsevier, 1988; vol 1: 143-59.

13 Haun F. Functional dissociation of the hemispheres using foveal visual input. Neuropsychologia 1978; 16: 725-34.

14 Früh H, Cook ND, Landis T, Regard M. Larger hemifield differences after bilateral stimuli presentation: a study on normal subjects and acallosal patients. Paper presented at the Twelfth European Workshop on Cognitive Neuropsychology. Bressanone, Italy, 1994.

15 Lansdell H. A sex difference in effect of temporal lobe neurosurgery on design preference. Nature 1962; 194: 852-4.

16 McGlone J. Sex differences in human brain asymmetry. Behavioral and Brain Sciences 1980; 3: 215-63.

17 De Lacoste-Utamsing C. Sexual dimorphism in the human corpus callosum. Science 1982; 216: 1431-2.

18 Witelson $\mathrm{S}$. Sex differences in neuroanatomical changes with aging. N Engl f Med 1991; 325: 211-2.

19 Kimura D, Harshman RA. Sex differences in brain organization for verbal and non-verbal functions. In: De Vries GD, De Bruin JPC, Uylings HBM, Corner MA, eds. Progress in brain research. Amsterdam: Elsevier, 1984: 423-41.

20 Bryden $M$. Measuring handedness with questionnaires. Neuropsychologia 1977; 15: 617-24.

21 Annett M. Left, right, hand and brain: the right shift theory. London: Laurence Erlbaum, 1985.

22 De Agostini M, Paré C, Goudot D, Dellatolas G. Manual preference and skill development in preschool children. Developmental Neuropsychology 1992; 8: 41-57. 